\title{
THEORIES OF OCCLUSION; AND THE SORPTION OF IODINE BY CARBON.
}

Captain J. W. McBain, D.Sc. (Bristol University), communicated a paper on "Theories of Occlusion : and the Sorption of Iodine by Carbon."

\section{PART I.}

The Prisfent Development of the Theory.

The occlusion of gases by metals comprises processes which are special instances of the general group of different phenomena known collectively as sorption.*

In the vast maj rity of cases the intermingling phenomena that constitute sorption have not been disentangled or even experimentally identified. From a theoretical point of view true adsorption, surface condensation, difference in concentration between the surface and the bulk of a liquid or gas, attracts by far the most attention. Yet the phenomena of absorrytion, or true solution in a solid, are also of interest ; and frequently the chemical reactions that ensue are of decisive importance, as, for example, in the behaviour of catalysts.

It may be asked why it should be considered of any practical importance to insist upon the distinction between these different factors. The answer is that the time relationships of adsorption and of absorption and again of chemical reaction are found to be widely different. The laws governing the amounts of gas or dissolved substance sorbed by these processes also differ; but this is of less importance, because when adsorption is present it sufficiently influences the net result to throw it into the form of an adsorption equation such as

$$
{ }_{m}^{x}=k p^{\frac{1}{n}}
$$

It is on account of these differences in time relationships that the author decided to make this his chief point when invited to contribute a note bearing on the theoretical and physical side of this discussion.

True adsorption is nearly instantaneous. Any lag can at present be accounted for by the time required for the dissipation of the heat evolved or the comparative inaccessibility of a portion of the surface of a porous adsorbing agent. On the other hand, absorption, or solid solution, may be reasonably expected to obey Fick's diffusion law, which requires that it should commence with a very high velocity for the first few moments, but rapidly fall off with time, and theoretically require infinite time for perfect equilibrium to be attained.

* The term "sorption" was introduced by the author, Phil. Mag., 1909 (6), 18; Zeit. physik. Chem., I909, 68, 47I) as a generic and non-hypothetical term for phenomena which frequently occur together; and it has been adopted by many authors since. 
Chemical reaction may present the most varied phenomena, particularly as reaction often proceeds vigorously in surface films as well as in solid solution. It is sometimes continuous, as in certain catalyses, and it is often overlaid by diffusion effects of the various reacting substances and their products. In the simplest case, where pure chemical compounds are formed, each of these exhibits a definite decomposition pressure, below which none is formed and at which formation is complete. All these phenomena are to a certain extent independent and may even be opposed to each other at a given moment. Reference may be made to previous papers for such behaviour in the case of the occlusion of hydrogen by carbon.*

I venture to assert that in most of the contributions to this subject no adequate attempt has been made to identify, much less isolate or evaluate, these separate factors. Too often mere prejudice, not experimental evidence, has decided an author to consider his particular case one of absorption or, more usually, of adsorption. Again, an author may be so intent upon studying adsorption that he ignores chemical reactions which may occur, such as the displacement of chemical equilibrium, metathesis, etc. In a few cases investigators have succeeded in simplifying their problem by using a liquid in place of a solid and by a suitable choice of chemicals. That, however, does not help for the purpose of the present discussion, where it is just the behaviour of solids that is in question.

The author wishes to make it quite clear that the standpoint here presented is by no means that all cases of sorption are of composite type ; but rather that until any particular case has received adequate experimental investigation it should be referred to as sorption and not given the more definite and therefore hypothetical name of adsorption, absorption, chemical reaction, etc. These names should be restricted to cases in which it has been proven to consist of only one of these.

\section{The Relative Importance of the Various Factors of Occlusion.}

The occurrence of absorption is necessarily conditioned by the possibility of measurable diffusion. This has been shown to occur, for example, in charcoal at $-180^{\circ}$, in palladium at ordinary temperatures, in gold and lead at moderately raised temperatures, and in steel at high temperatures. It is hastened by rise of temperature, which may thus render it practically appreciable even where the true solukility may be lessened

On the other hand, adsorption rapidly diminishes with rise of temperature. It is also conditioned by the extent of surface, which is often small in the case of metals. The surface of metallic grains embedded in the interior of an alloy is only accessible through diffusion.

For these reasons it seems plausible to predict that occlusion phenomena will be largely those of diffusion, of absorption, not so much of adsorption. Chemical reaction will also play a prominent part.

These two phenomena, chemical reaction and solid solution, present

* For further evidence for the case of hydrogen and carbon see Firth (Zeit. physik. Chem., 1914, 86, 294). Again, the careful investigation of the occlusion of hydrogen by palladium has been shown to belong to this composite type (Holt, Edgar, and Firth, Zeit. physik. Chem., r913, 82, 5I 3 and 820; Sieverts, ibid., 191 4, 88, 103; Wolf, ibid., I914, 87, 575; Holt, Proc. Roy. Soc., A I9I4, 90, 226). Georgievics has published much evidence in the cases of dissolved substances sorbed by textiles; Langmuir is led to the same conclusion in discussing the time relationships of the sorption of water vapour by glass or silica, etc. 
but little mystery in themselves and sufficient criteria are available to identify them by experiment in any concrete case. The seni-quantitative behaviour of diffusion is also easily followed, although its quantitative mathematical expression is often complicated.

One further point in the case of metals subjected to heat treatment is that processes which have occurred at higher temperatures sometimes become fixed on cooling on account of diffusion being practically inhibited at lower temperatures, and thus not allowing of any redistribution at the lower temperatures.

\section{Theories of Adsorption Proper (Surface Condensation).}

Adsorption is essentially a surface tension phenomenon, sometimes influenced also by electro-capillary effects. Such condensation in the surface separating any two homogeneous phases will always occur whenever the surface tension or any other potential energy in the surface is lessened thereby, in accordance with Wolfgang Ostwald's extension of Willard Gibb's classical equation. Unfortunately, nothing is known with regard to the quantitative values of the surface tension of solids, and hence no direct experimental comparison has been made here such as was carried out for liquid surfaces by Lewis, Milner, Donnan and Barker.

Failing then a direct thermodynamical treatment, the atomic theory has been resorted to in order to obtain a picture of the mechanism involved in adsorption, as well as its relation to solid solution and chemical reaction. The most notable contributions to these conceptions have been made by Haber (I), Harkins (2), and Langmuir (3), inspired by the work of Bragg and Lawe on the X-ray structure of crystals.

Langmuir considers that every atom within a solid is tied to an equilibrium point in a space lattice, but that it is attached to all neighbouring atoms by primary or secondary chemical valency. Thus, a crystal would constitute a single large molecule, held together by purely chemical forces. The layer of atoms constituting the surface would thus present unbalanced and unsatisfied valencies on their outer side. This would be the origin of surface energy ; and surface tension, like cohesion, would be explained as due to purely chemical forces.

Adsorption is regarded by Langmuir as being the attachment of outside molecules or atoms to the exposed free valencies of the atoms comprising the surface layer. Thus adsorption is regarded as due to purely chemical forces. Harkin's chief contribution, from our present standpoint, is that all molecules in the surface layer are so oriented as to minimize surface energy by exposing the least active part of the molecule; for example, in a fatty acid the carbonyl group points inwards and the terminal $\mathrm{CH}_{3}$ group outwards. Langmuir shares this view.

The process suggested for the way in which adsorption films are formed is interesting, and comes much more within the reach of experimental control. Nearly every molecule which happens to strike a solid surface is retained, being condensed and held instead of reflected. This is the case at all temperatures. The surface film so formed tends to be a continuation of the space lattice of the solid. On the other hand, evaporation of such a film is going on as an independent process, being so rapid at high temperatures as to keep the surface practically clean. Adsorption is a direct consequence of the time lag between condensation and evaporation, and is the result of the kinetic equilibrium. The film 
formed may cover only a fraction of the surface, and it is usually only one atom or molecule deep. Experimental evidence is cited (4) for the statement that most molecules striking a surface are not reflected, in addition to Langmuir's experience with the formation of shadows during the evaporation of metallic filaments in a high vacuum.

This brief reference cannot possibly do justice to J.angmuir's interesting monographs (his papers cover about 250 pages). Its purpose is to call attention to these interesting and clear-cut views, which appear to be in accordance with the known facts, although they differ widely from other views current in the literature of sorption. They stimulate to experiment to an unusual extent for this class of paper, and they are illustrated by a wide range of phenomena. It is quite obvious that adsorption proper will have to be elucidated in the light of experiments made upon known, definite, preferably plane, surfaces. From a practical standpoint, on the other hand, there is still great need for the further experimental investigation of sorption by carbon, in spite of the great uncertainty as to its real surface and porosity.

The important feature, in its direct reference to the present discussion, is the insistence upon the fact that among the scanty experimental evidence so far available there is no evidence for the existence of films more than one molecule deep except where the surface is porous and where the gas adsorbed is a nearly saturated vapour. If a surface is porous, as in the case of charcoal, or of a metal covered with a porous film of oxide or tarnish, the real surface is immensely magnified, and the adsorbed film appears to be many molecules thick if calculated merely for the apparent external area. A really clean polished metallic surface is not considered to be porous in this sense, any more than is that of glass, for example. Experiments by S. P. Sweetser are described, demonstiating the formation of films not more than one molecule teep in the adsorption of gases by mica, glass, and platinum over a wide range of temperature, but an exception is to be found in the condensation of thicker films on glass by cadmium vapour. Other instances of monomolecular films in the case of oils and fatty acids and water are given by the striking experiments of Marcelin and Devaux (5), which lead to a determination of the orientation and actual shape of the molecules in the adsorbed film.

With regard to the empirical exponential adsorption formula quoted above. Langmuir agrees that at very low pressures there is a deviation in that the amount of adsorption becomes nearly proportional to the pressure. This result has been obtained by many experimenters with gases. Litorff and Miss Homfray obtained isolated values for $\mathrm{I} / n$ which approach 2 ; Arrhenius (Io) attributes this to experimental error, which must, if so, be very great. In fact, the lack of exact experiment, including the influence of time, is the chief difficulty in elucidating the theory of these phenomena. Arrhenius also points out that the saturation value appears to be independent of the temperature, and that the value of the coefficient $\mathrm{r} / n$ depends only on the nearness of the amount sorbed to this saturation value, both lending support to Langmuir's suggestion.

The behaviour of gases at low pressures is, however, quite different from that of solutions. For example, Trümpler (6) found for very dilute solutions of fluorescein containing only from o.00ooo I to o.00I gram per litre, that the adsorption part of the sorption he observed was proportional to only the o.I8th power of the concentration. In the case of solutions of acetic acid in alcohol (7), this purely empirical exponential formula expresses the results over a range of concentration of 23,000 -fold. 
At higher pressures the amount of gas sorbed by carbon appears to approach a maximum (quite apart, of course, from the phenomena met with when nearly saturated vapours are employed), although further experiments are desirable. This is quite different in kind from the superficially similar appearance of maxima of sorption in the cases of solutions in which the solvent too is sorbed. Here the apparent maximum (apart from the formation of colloidal film) is due to the method of measurement, which in concentrations where relatively more solvent than solute is sorbed produces so-called negative sorption. The nearest analogy with gases is to be found in the experiments of Marc (8) on the adsorption of dyes by crystal surfaces. In this case, however, the exponential formula holds right up to the constant saturation value.

The experiments described in Part II refer to solutions, but the results illustrate also some of the features observed with gases which are referred to above. It has been impossible on account of military duty for the author to undertake the direct experiments which he would have liked to have carried out.

\section{PART II.}

\section{FXPeriments ON tHe SORPtion OF IODine By Carbon EXTENDing over Pertods of Many Years.}

In 1907, my colleague, Dr. O. C. M. Davis, communicated from this laboratory the first experimental results to show that adsorption may be accompanied by absorption, that in addition to a rapid condensation on the surface there is a slow diffusion which goes on for long periods of time. This behaviour is illustrated by a table of data taken from Table XV of his publication (9), but continued in the last two lines by analyses of experiments set up by him in 1907 and now, after standing at room temperature for eleven years, completed by Miss M. E. Laing. $a$ is the original amount of iodine ( 1.2685 grams) in Ioo c.c. of the toluene solution; $a-x$ is the number of grams of iodine left in solution after shaking with the carbon; $m$ is the weight of carbon (2 grams) shaken with Ioo c.c. of solution; $x / m$ has therefore its usual significance of grams of iodine sorbed by one gram of charcoal.

TABLE I.

Sorption by Carbon of Iodine dissolved in Toluene.

\begin{tabular}{c|c|c}
\hline Time. & $x / m$. & $a-x$. \\
\hline & grm. & grm. \\
5 min. & 0.229 & 0.809 \\
I5 ", & 0.237 & 0.793 \\
30 , & 0.245 & 0.777 \\
I hour & 0.265 & 0.736 \\
2 hours & 0.293 & 0.682 \\
5 ", & 0.299 & 0.669 \\
24 ", & 0.315 & 0.637 \\
5 days & 0.327 & 0.613 \\
24 ", & 0.379 & 0.509 \\
I I years & 0.481 & 0.307 \\
I I ," & 0.509 & 0.250 \\
\hline
\end{tabular}


Some of the original solution of iodine in toluene was extracted with water after two years and the aqueous extract was tested with silver nitrate. There was no precipitate, showing that no hydriodic acid had been formed by chemical reaction.

Stili more striking is the fact that after eleven years the aqueous extract of the toluene solution, and even of the carbon which had been sealed up with it, gave only a slight opalescence on testing with silver nitrate. Thus the slow diffusion cannot be ascribed to chemical interaction. Similar experiments on benzene solutions of iodine are given in Table II. Here again the aqueous extract of the benzene solution and of the carbon in contact with it for eleven years gave no reaction with silver nitrate.

TABLE II.

Sorption by Carbon of Iodine dissolied in Benzene.

\begin{tabular}{c|c|c}
\hline Time. & $x / m$. & $a-x$. \\
\hline & grm. & grm. \\
3 days & 0.328 & 0.611 \\
Io , , & 0.339 & 0.589 \\
I I years & 0.386 & 0.495 \\
& 0.561 & 0.146 \\
\hline
\end{tabular}

In both cases it is evident that the amount of iodine sorbed at the end of 24 hours is only about two-thirds of that finally taken up from the toluene or benzene solution. This is in spite of the fact that the sorbing power of carbon is slowly impaired with age. Furthermore, equilibrium experiments communicated by Davis similar to those described below (Table VI) show that during all this time there has been equilibrium between the adsorbed iodine and the toluene or benzene solution. Hence, as the author showed in the case of hydrogen and carbon,* considerable iodine must have been transferred during this time from the surface where it was initially deposited, and has been carried into the interior of the carbon. In the case of the toluene experiments, the amount of iodine so transferred after 24 hours was about 0.073 grams, in addition to the 0.180 grams coming from the toluene, making a total of 0.253 grams in the interior and $0 \cdot 24$ grams where it was deposited during the first day. Thus the diffusion refers to over half of the total iodine finally sorbed. This deduction is based on the assumption that the whole of the sorption occurring during the first 24 hours was adsorption. It will be recollected that according to the known laws of diffusion the first stage of absorption takes place with great rapidity, falling off very rapidly indeed after the first few moments; hence solid solution will amount to more than the above figures imply.

\section{Experiments on Benzene Solutions by H. F. Baggs $\dagger$ and Miss M. E. Watson.}

In I9I4, Dora Schmidt-Walter (II) controverted the results of Davis as well as McBain. Her work has been quoted by several careless
* Loc. cit.
$\dagger$ Died of wounds, January 1917 . 


\section{THEORIES OF OCCLUSION ; AND THE SORPTION OF}

readers (I2) as having disproven the work of Davis on benzene and iodine, without their having even noticed the extraordinary fact that her paper contains no numerical data whatever with regard to these chemicals, but a mere reiterated statement that they did not show any effect of time. If this were true of any one solvent it would invalidate our work on carbon, since we have supposed that the diffusion occurs within the carbon itself, a reasonable assumption in view of the magnitude of the effect and of its occurrence in the case of several gases where no solvent is employed.

The experiments in Table III were carried out with animal carbon obtained in I9I4 from the same source as Dora Schmidt-Walter's and treated exactly as she described. We, however, took the precaution of sealing up each tube, wrapping it in lead foil and shaking it in a shaking machine in a thermostat at $25^{\circ}$. Each result is the mean of two independent duplicate experiments.

TABLE III.

Sorption by Animal Carbon of Iodine from Solution in Benzene.

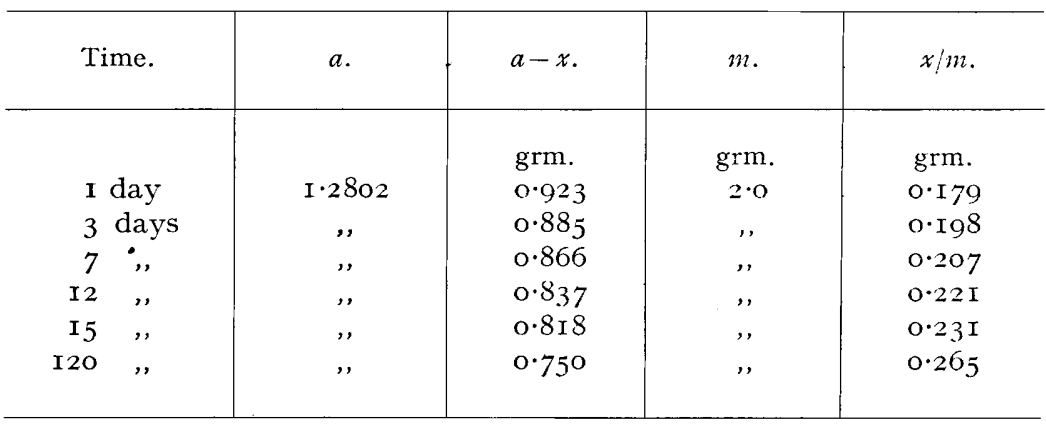

These results confirm those of Davis.

The experiments in Table IV were carried out with sugar carbon, since this must be free from many of the impurities remaining in animal carbon even after careful treatment.

TABle IV.

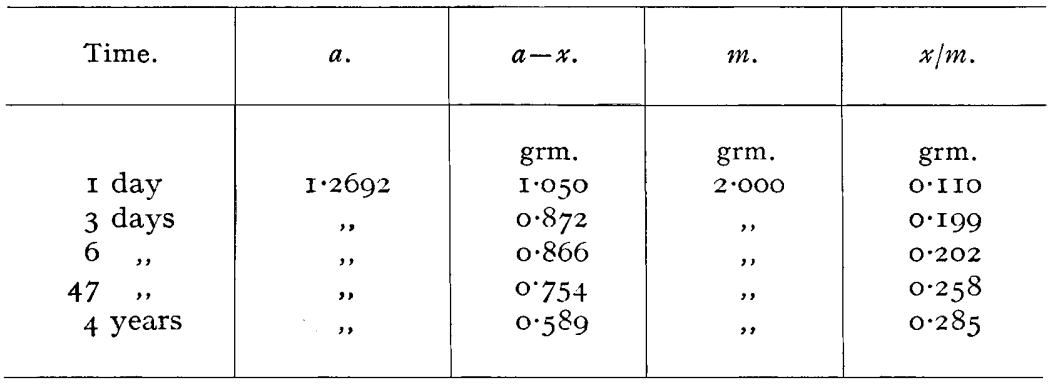

The four-year experiments in quadruplicate. Titrations $5.55,5.65$, $5 \cdot 575,5 \cdot 65$ c.c.

Here again tests with silver nitrate revealed no chemical reaction after four years. 
Experiments by Miss M. E. Laing on the Sorption by Sugar Carbon of Iodine dissolved in Carbon Tetrachloride.

It appears to be impossible to choose a solution such that chemical reaction is obviously excluded during sorption by carbon, but as the possibilities are at a minimum in this case it was chosen for study.

The sugar carbon was dried by placing it in a silica flask surrounded by the flame of a blowpipe and evacuating the red-hot flask for some hours by a Toepler pump. The carbon tetrachloride was dried with calcium chloride and distilled. The iodine was sublimed in the ordinary way from potassium iodide, resublimed and dried in a desiccator. The experiments, like all others in this communication, where not specially noted, were carried out at $25^{\circ}$, in the dark, in sealed tubes. Titrations were made with decinormal sodium thiosulphate, using calibrated pipettes. Actually 1.4 .83 c.c of original solution were placed in the sealed tube with the corresponding weight of carbon. On opening the tube, the solution was sucked up into a ro.o9 c.c. pipette through a small tube packed with filter paper, and the first portion coming through was thrown away to eliminate any effect of sorption by the filter paper. (The duplicate was filtered through the same plug of filter paper.) It was often difficult to be sure that no carbon got through, as it is in such fine powder.

The results are given in Table $V$, where the burette readings are given for the duplicate experiments as a guide to the degree of experimental error.

TABLE V.

Sorption by Carbon of Iodine from Carbon Tetrachlorite.

\begin{tabular}{|c|c|c|c|c|c|}
\hline Time. & a. & $m$ & Titrations. & $a-x$. & $x / m$. \\
\hline 24 hours & $\mathrm{I} \cdot 074$ & $3 \cdot 37 \mathrm{I}$ & 0.40 c.c. & $0.04 \mathrm{I}$ & 0.307 \\
\hline $48 \quad$, &, & ," & 0.12 and 0.13 & 0.013 & 0.315 \\
\hline 3 days & ," & ,. & $0.09,, 0.08$ & 0.009 & 0.316 \\
\hline $7 \quad$, & ," & ," & $0.07,, 0.08$ & 0.008 & $0 \cdot 316$ \\
\hline 28, & ", & ", & $0.04,, 0.04$ & 0.004 & 0.317 \\
\hline 24 hours & $I \cdot 269$ & $I \cdot 686$ & 34.86 c.c. $*$ & 0.454 & 0.484 \\
\hline $4^{8} \quad$, & ," & ", & $3 \mathrm{I} \cdot 29$ and $3 \mathrm{I} \cdot 52$ & $0 \cdot 394$ & 0.518 \\
\hline 7 days & , & " & $29 \cdot 78,, \quad 29 \cdot 76$ & 0.374 & 0.522 \\
\hline 24 hours & $I \cdot 09 I$ & I.OII & 5.56 and 5.52 & 0.567 & 0.518 \\
\hline $4^{8} \quad$, & ," & ", & $5 \cdot 28,, \quad 5 \cdot 32$ & 0.543 & $0.54^{2}$ \\
\hline 3 days & ," & ,", & $5.03, \quad 5.04$ & $0.5^{16}$ & 0.569 \\
\hline 23, & ," & ", & $4 \cdot 92,, 496$ & 0.5 II & 0.574 \\
\hline 24 hours & $I \cdot 25^{8}$ & $0.33 \overline{7}$ & $8 \cdot 42$ and $8 \cdot 44$ & $x \cdot 060$ & 0.587 \\
\hline $4^{8} \quad$, & ", & ," & $8 \cdot 32,, 8 \cdot 36$ & I.049 & 0.619 \\
\hline 3 days & ,", & ," & $8.29,, \quad 8 \cdot 35$ & $I \cdot 0_{4} 6$ & 0.628 \\
\hline I7 & ," & ", & $8.15,, 8.25$ & $\mathrm{x} \cdot 03 \mathrm{I}$ & 0.673 \\
\hline
\end{tabular}

The data of Table $\mathrm{V}$ cannot be discussed to advantage until their remarkable significance is brought out in the light of equilibrium experi-

* Titrated with $\mathrm{N} /$ Ioo thiosulphate. 


\section{IO THEORIES OF OCCLLSION; AND THE SORPTION OF}

ments such as those of Table VI. Two experiments carried out in duplicate are described. In A, ro.09 c.c. of about $\mathrm{N} / 5$ solution of iodine in carbon tetrachloride and 10.22 c.c. of pure solvent were pipetted into a tube together with $O \cdot 5_{5}$ oo grams of carbon and the tube seaied, shaken for 24 hours at $25^{\circ}$ and titrated. In $\mathrm{B}$, the same materials and pipettes were used, but at first only the ro.og c.c. of $\mathrm{N} / 5$ iodine was added, and the tube sealed. It was shaken carefully so as not to wet the drawn-out end, and after four hours it was opened, the ro.22 c.c. of pure solvent added, and the tube resealed and analysed after a further 20 hours.

TABLE VI.

True Equilibrium between Carbon and Iodine dissolved in $\mathrm{CCl}_{+}$ at $2+$ Hours.

\begin{tabular}{c|c|c|c}
\hline Experiment. & Titrations. & $a-x$. & $x / m$. \\
\hline A & $6.9 \mathrm{I}$ and 6.92 & 0.8690 & 0.4929 \\
B & $6.9 \mathrm{I}, 6.95$ & $0.87 \mathrm{IO}$ & $0.490 \mathrm{I}$ \\
\hline
\end{tabular}

Thus there is a true reversible equilibrium attainable from both sides in the sorption of iodine from carbon tetrachloride at 24 hours. This is in spite of the fact, demonstrated in Tabie $V$, that the same carbon which has thus given up any excess of iodine beyond this 24 hours' value will now proceed to take up further iodine for months and years.

Similar experiments have been carried out by a number of authors, and they all give the same result: equilibrium at say 24 hours. It would be interesting to study the effect of altering the time-to a minimum, for example; but such experiments have not been published. The equilibrium would appear to be attained more rapidly from the side of higher concentration, in accordance with the conception of incipient solid solution in the superficial layers ( $\mathrm{r}_{3}$ ). It appears impossible, in the face of such evidence as the above, to deny that surface condensation takes place rapidly and reversibly in a short time, with regard to the available surface. Further, that diffusion of some sort subsequently takes place. This diffusion lasts over months and years and involves a fraction, sometimes more than half, of the total sorbed iodine. Some of the initially deposited iodine is afterwards transferred to places thus proven to be accessible only through diffusion.

It would seem natural to conclude that this final depository is within the substance of the carbon itself, in other words, solid solution. However, a study of Table $V$ reveals that the quantities involved in the diffusion do not bear the expected proportionality to the concentrations of the carbon tetrachloride solutions. Unfortunately, the table, and in particular the data of the first five lines, will not bear the weight of elaborate mathematical analysis, chiefly because the adsorption is so great in comparison with the diffusion observed. More extensive data are urgently needed, especially dealing with the extremes of concentration. As the data stand, the diffusion is much more nearly related to the value of $x / m$ than to that of $a-x$, which would indicate that at least a part of this unquestionable diffusion must be engaged in transporting iodine adsorbed on the externally accessible surfaces to other 
interior surfaces, where it is deposited in a state of adsorption-unless indeed we are to believe that the distribution law is not applicable to solid solution either while diffusion is proceeding or to the solution near the surface. How great the actual amount of solid solution, of absorption, remains, is still uncertain on account of the possibility of these interior surfaces in carbon particles.

At 24 hours the sorption is accurately represented by the formula $\frac{x}{m}=0 .{ }_{5} 80(a-x)^{0.1995}$, which is a straight line on the logarithmic diagram. With lapse of time the graph becomes curved, so that the exponential formula is less applicable. Presumably this may be ascribed to the development of solid solution.

It should be remembered that the carbon is an impalpable powder, and that experiments in which carbon has been freed from gases and the sorption carried out in vacuo give the same results ( $\left.\mathrm{I}_{4}\right)$. Further, similar phenomena are met with in the case of some gases with carbon, where no liquid solvent is present, although no experiments involving great periods of time have been carried out. It is obvious that a most interesting field for further experiment has been opened out.

\section{SUMMARY.}

I. It is once more emphasized that sorption phenomena should not receive clear-cut designations as adsorption and absorption (solid solution), etc., until the experiments have arequately shown that the particular case is a pure type of only one of these factors, which are known in a number of cases to occur simultaneously.

2. Attention is drawn to Langmuir's hypothetical explanations of all these as chemical phenonena, in which he has crystallized out views shared by a number of authors.

3. Fxperiments on the sorption of iodine by carbon, extending over periods up to eleven years, are described. The long-continued diffusion following on the rapid surfacc condensation involves in the case of aninial carbon more than half of the total iodine sorbed.

\section{REFTRFACLS.}

r. HABER : Zeit. Elehtroshem, I91.i, 20, 524.

2. Harisins, Davies, and Clark: J. Amcr. Chom. Soc., I9l $7,39,54$ I.

3. Langmulr: Phys. Rev., Igr5, 6, 79; ibid., I9x6, 8, r49; Met. Chem. Eng., 1916, 15, 468; I. Amer. Chem. Soc., IGI6, 38, 2221 ; ibid., r9r7, 39, 18.48; ibid., 1918, 40, $136 \mathrm{r}$.

4. Herz: Amm. Physik, 1882, 17, 177; Badle: Trans. Roy. Soc., 1879, 170, 249; Wood, Phil. Mag., I915, 30, 300; Millikan: Ann. Physik., I911, 35, 389: Langmuir: Physikal. Zeit., 191., 1273; Phys. Rev., 191.4, 2, 450 .

5. Marcelin: Ann. Phy'sik., I9I4, I, r9: Devaux: Swithsonian Inst.'s Annual Report, 19r4, 26r ; see also RayleIgH: Phil. Mag., I899, 48, 331; Labrouste: C. R., I9I4, 158, 627; Hardy: Proc. Roy. Soc., I9I2, 86, A 6Io.

6. TRÜMPLER: Kolloid-Ziit., 1914, 15, 10.

7. Gustavson: Zeit. physik. Chem., I916, 91, 385.

8. Marc: Zeit. physik. Chem., I9I r, 75, 7ro.

9. DAvis: Trans. Chem. Soc., I907, 91, I674. 
Io. Arrhenius: Medd. K. Vetenkapsakad. Nobel-institut, I9II, 2, No. 7 .

I1. Schmidt-Walter: Kolloid-Zeit., I9I4, I4, 2.22.

12. For example, TAYLOR: Chemistry of Colloids, I9I5, p. 28I ; but see Wo. Ostwald: Theoretical and Applied Colloid Chemistry, I917, p. II 7 .

13. McBain: Zeit. physik. Chem., I909, 68, 47 I.

I4. Davis: see 9, and Schmidt-Walter, see it. 